\title{
CrystEngComm
}

\section{Microwave-assisted synthesis of icosahedral nickel nanocrystals}

\author{
Keith P. Donegan, ${ }^{a}$ Jeffrey F. Godsell, ${ }^{c}$ Joseph M. Tobin,${ }^{a b}$ Justin P. O'Byrne, ${ }^{a b}$ David J. Otway, ${ }^{a}$ \\ Michael A. Morris, ${ }^{a b}$ Saibal Roy ${ }^{c}$ and Justin D. Holmes ${ }^{* a b}$
}

\author{
Received 22nd October 2010, Accepted 30th November 2010 \\ DOI: $10.1039 / \mathrm{c0ce00759e}$
}

\begin{abstract}
Nickel nanocrystals with icosahedral morphologies have been successfully synthesised using a microwave-assisted irradiation method. Nickel acetylacetonate was used as the metal precursor, while sodium formate and trioctylphosphine oxide were employed as the reducing agent and capping ligands, respectively. The nanocrystals, with a mean diameter of $237 \pm 43 \mathrm{~nm}$, exhibited enhanced ferromagnetic behaviour at room temperature compared to bulk nickel, with coercivities of up to 164 Oe and saturation magnetisation values of up to $46 \mathrm{emu} \mathrm{g}^{-1}$, due to their icosahedral morphologies.
\end{abstract}

\section{Introduction}

Magnetic nanoparticles have potential use as active components in a number of applications, such as high density magnetic storage devices, contrast enhancement in magnetic resonance imaging, biotechnology and catalysis, ${ }^{1-4}$ owing to their size dependent physical and chemical properties. ${ }^{5}$ As particle size is reduced down to the nanometre-length scale, novel optical, electronic, catalytic and magnetic properties arise as the ratio of surface to bulk atoms increases. ${ }^{6-9}$ For magnetic nanoparticles, the critical volume is reached when the energy of the particles can no longer support the existence of domain walls and thus become a single, magnetic domain. Hence, the magnetic properties of these nanoparticles and any future potential applications are dictated by their size and morphology.

Metallic nanostructures such as rods, wires, sheets and plates have been synthesised in an attempt to improve the magnetic properties of nanocrystals. ${ }^{10-13}$ These nanostructures have demonstrated increased magnetic and catalytic properties owing to their high shape anisotropy compared to their spherical counterparts. For example, nanoplatelets synthesised by Xu et $a l$. were found to have coercivities of up to 120 Oe at room temperature. ${ }^{14}$ Transition metal nanostructures of shapes other than the most thermodynamically favourable, i.e. spherical, are notoriously difficult to synthesise as the reaction kinetics are often difficult to control. Highly faceted multiple twinned nanocrystals of platinum, palladium, gold and silver have recently been reported, ${ }^{15-18}$ but the synthesis of nickel particles with similar morphologies has not been investigated.

${ }^{a}$ Materials and Supercritical Fluids Group, Department of Chemistry and the Tyndall National Institute, University College Cork, Cork, Ireland. E-mail: j.holmes@ucc.ie; Fax: +353 (0)21 4274097; Tel: +353 (0)21 4903608

${ }^{b}$ Centre for Research on Adaptive Nanostructures and Nanodevices (CRANN), Trinity College Dublin, Dublin 2, Ireland

${ }^{c}$ Microsystems Centre, Tyndall National Institute, University College Cork, Lee Maltings, Cork, Ireland
According to Wulff's theorem, the equilibrium shape of a single crystal of a face-centred cubic (fcc) metal is the Wulff polyhedron (a truncated octahedron). ${ }^{19}$ Non-equilibrium shapes are often found in nanoparticles due to a large proportion of edge atoms and non-negligible edge energies resulting in stable alternative structures. ${ }^{20}$ The presence of twinning defects is one of the major causes of non-equilibrium shapes, especially in fcc crystals which have low twin boundary energies. ${ }^{21}$ The presence of twin defects during the nucleation and growth stage could potentially result in the synthesis of decahedral and icosahedral nanocrystals which have a total free energy less than that of the Wulff polyhedron. A number of methods have been utilised for synthesising transition metal nanoparticles including alcohol reduction techniques, thermal decomposition of organometallic precursors, electrochemical methods and the reduction of metal salts. ${ }^{22-25}$

Microwave irradiation has also been utilised to generate novel materials. ${ }^{26}$ Precursors and their reaction intermediates are thought to have different dielectric constants along the same reaction paths. Microwave irradiation overcomes these differences by selectively coupling to intermediates in their transition states. Microwave irradiation as a heat source has numerous advantages over conventional heating methods. The irradiation first couples with the material and is then absorbed, resulting in the conversion of electromagnetic energy into thermal energy. Heat is thus generated from inside the material, in contrast with traditional heating methods where heat is transferred from the outside to the inside. This internal heating mechanism results in both reduced reaction times and energy costs. Selective formation of one phase over another often occurs, resulting in the synthesis of new materials. ${ }^{27,28} \mathrm{~A}$ possible explanation for these microwave effects is the generation of localised high temperatures or "hot-spots" at the reaction sites, which enhance the reaction rates in a manner similar to that of ultrasonic irradiation. ${ }^{29}$ Microwave irradiation has been employed as a heat source in various fields, such as in the synthesis of mesoporous silica, catalysis and in the synthesis of metal and metal oxide 
nanoparticles. ${ }^{30-33}$ In this paper, we report on the synthesis of novel, highly faceted, multiple twinned nanocrystals of nickel using microwave irradiation as a heat source.

\section{Experimental}

Nickel acetylacetonate, $n$-trioctylphosphine oxide, sodium formate, ethylene glycol and other chemicals were all analytical grade and used without further purification. In a typical synthesis, nickel acetylacetonate $\left(3 \times 10^{-4} \mathrm{~mol}\right)$, sodium formate $\left(1.5 \times 10^{-4} \mathrm{~mol}\right)$ and $n$-trioctylphosphine $\left(3 \times 10^{-4} \mathrm{~mol}\right)$ were dissolved in $6 \mathrm{ml}$ of ethylene glycol in a $10 \mathrm{ml}$ microwavable tube. The reaction was carried out in a CEM Labmate $\AA$ microwave oven and heated for varying time periods with $91.6 \%(275 \mathrm{~W})$ of the total output $(300 \mathrm{~W})$. A black precipitate was obtained and this was separated from the reaction solution and washed a number of times with ethanol to remove any excess surfactants.

The size and morphology of the particles were determined by scanning electron microscopy (SEM) on an FEI Inspect F with an operating voltage of between 5 and $10 \mathrm{kV}$ and transmission electron microscopy (TEM) on a JEOL 200 and a JEOL 2100, both operating at $200 \mathrm{kV}$. The samples for microscopy were prepared by diluting the solution in ethanol and then dropping this dispersion of nanoparticles onto a carbon-coated copper grid for TEM analysis or a silicon wafer for SEM analysis. Energy dispersive X-ray (EDX) spectroscopy was carried out on an Oxford Instruments detector attached to a JEOL JSM-5510 scanning electron microscope. For analysis, solutions of dry nanoparticles were dispersed onto sticky carbon tape.

X-Ray diffraction (XRD) patterns were determined on a Phillips Xpert X-ray diffractometer with graphite monochromatised $\mathrm{Cu} \mathrm{K} \alpha$ radiation $(\alpha=1.54178 \AA$ ). To prepare the sample for analysis, a dilute solution of the nanoparticles in ethanol was dispersed onto a glass slide and allowed to dry. A scan rate of $0.025^{\circ} \mathrm{s}^{-1}$ was applied to record the pattern in the range $2 \theta=20-85^{\circ}$.

The magnetic properties of the particles were measured using a Quantum Design MPMS-XL5 superconducting quantum interference device (SQUID). The powder samples were placed within a polyamide capsule and characterised with an applied field range of $\pm 3 \mathrm{~T}$ and over a temperature range of 300 to $5 \mathrm{~K}$.

\section{Results and discussion}

Fig. 1 displays a microwave reaction profile for the synthesis of the nickel nanocrystals using nickel acetylacetonate as the starting precursor, with a microwave hold time of 10 minutes. The time, temperature and power are shown for a given reaction profile. The reactions were carried out at a power of $275 \mathrm{~W}$ and a maximum temperature of approximately $185^{\circ} \mathrm{C}$. The temperature localised around metal ions in solution is expected to have a higher temperature than that of the bulk solution. These "hotspots" have been proven to have an effect on the overall product formation. ${ }^{34}$

Fig. 2 shows SEM images of nickel nanocrystals synthesised from a microwave reaction with a hold time of 10 minutes. A higher-magnification SEM image (Fig. 2(b)) revealed that the particles were icosahedral in shape.

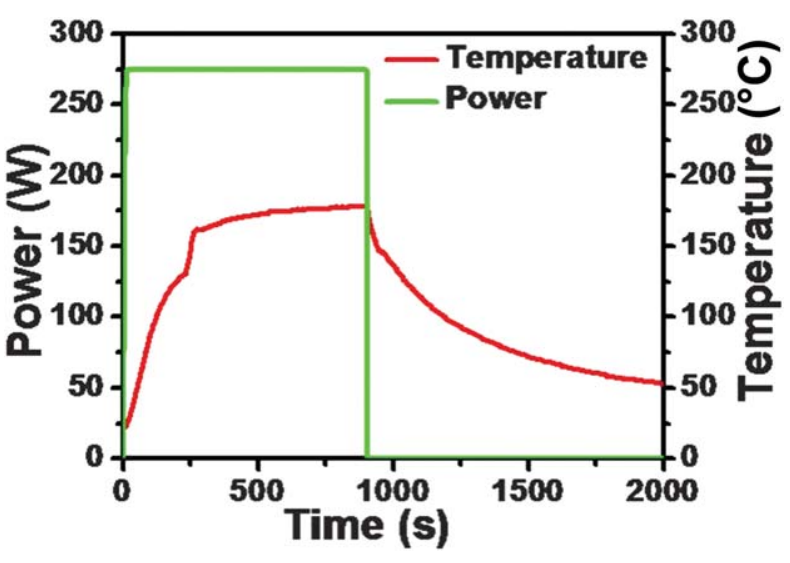

Fig. 1 Temperature, time and power (W) profiles of a typical nickel nanocrystal synthesis experiment using nickel acetylacetonate as the metal precursor, with a microwave hold time of 10 minutes.

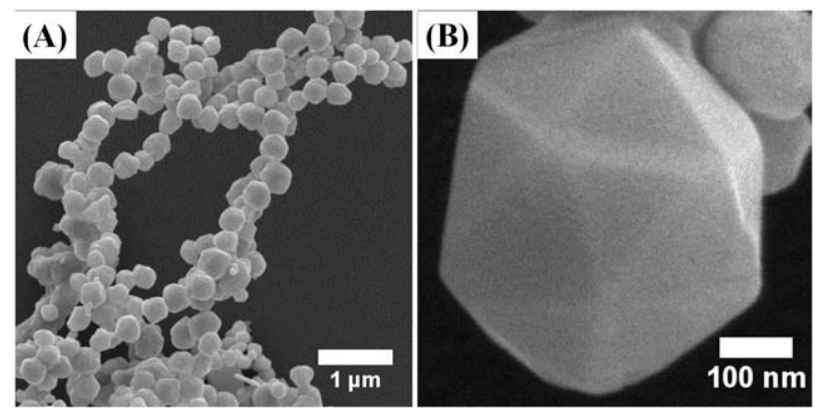

Fig. 2 (a) SEM images of nickel nanocrystals prepared using nickel acetylacetonate as the metal precursor with a microwave hold time of 10 minutes. (b) High magnification SEM image of the nickel nanocrystals shown in (a).

Fig. 3 displays TEM images of a typical icosahedral nanocrystal with an average edge length of approximately $77 \mathrm{~nm}$. Sixfold twinning of the nanocrystal is observed, with the twinning planes radiating out from the central point of the nanocrystal. The outlines in Fig. 3(a) represent the areas shown in Fig. 3(b) and (d). In Fig. 3(b), a TEM image of a corner of the nickel nanocrystal is displayed and a twinning boundary is observed. This is typically the thinnest part of the nanocrystal, allowing the electron beam to pass through the crystal and observation of the lattice spacings of the different crystal planes. While some lattice spacings are located and labelled in Fig. 3(c), these lattice spacings are only approximate measurements owing to the sloping faces of the nanocrystal, i.e. the TEM beam is not perpendicular to the crystal face so more accurate lattice spacing measurements were taken from X-ray diffractograms of the nickel nanocrystals. The $\{111\}$ crystal face of the nanocrystal is identified in Fig. 3(d) and the Fast Fourier Transform used to identify this plane is shown in the inset of this figure.

Fig. 4 shows an X-ray diffraction pattern of nickel nanocrystals deposited on a glass substrate. These crystals were synthesised using a hold time of 10 minutes. The three peaks at $43.8^{\circ}$, $51.2^{\circ}$ and $75.8^{\circ}$ can be indexed to the (111), (200) and (220) diffraction planes, respectively, from a face-centred cubic (fcc) structure. The XRD pattern of the nickel nanocrystals reveals no 

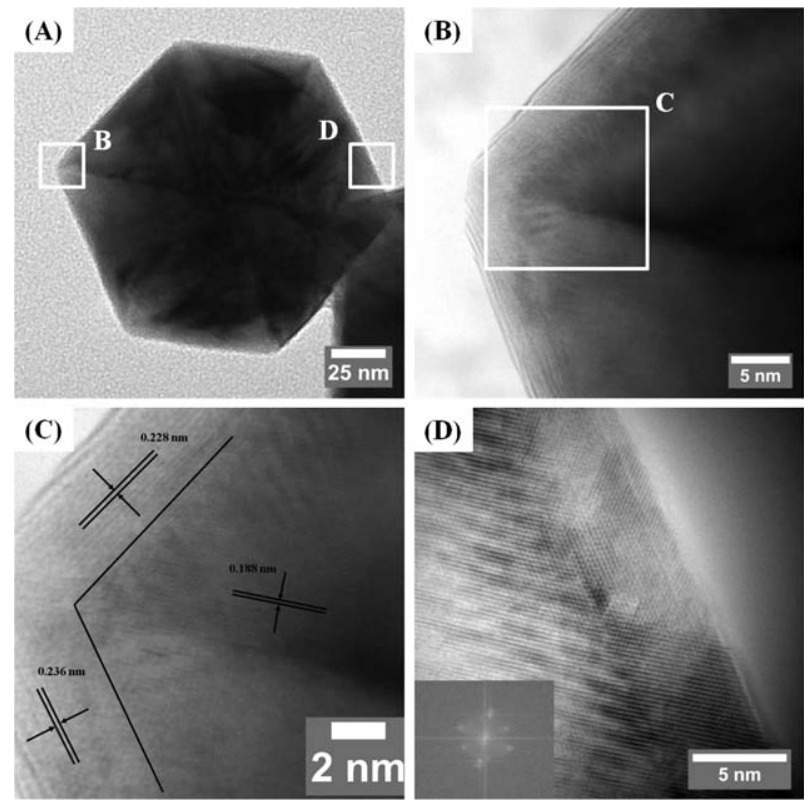

Fig. 3 TEM images of a single, icosahedral nickel nanocrystal, synthesised using nickel acetylacetonate as the metal precursor with a microwave hold time of 10 minutes. The outlines in (a) represent the areas shown in (b) and (d) while the outline in (b) represents the area shown in (c). The Fast Fourier Transformation (FTT) pattern of the nickel nanocrystal is shown in the inset of (d).

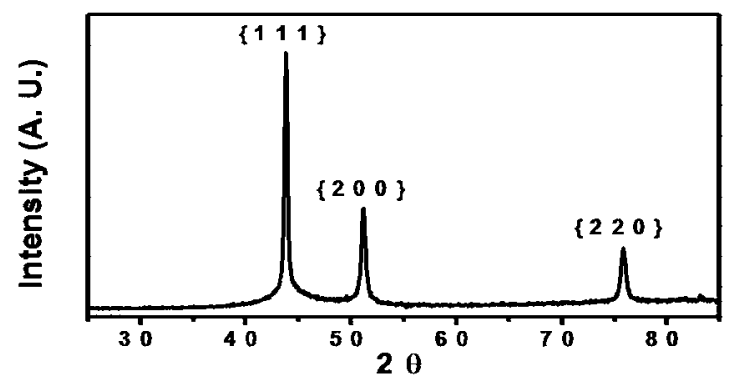

Fig. 4 X-Ray diffractogram of nickel nanocrystals prepared using nickel acetylacetonate as the metal precursor, with a microwave hold time of 10 $\min$.

other distinct diffraction peaks, indicating a high level of crystallinity and purity of the particles prepared. The XRD pattern exhibits a strong (111) diffraction peak and the ratio of intensity of the (111) to (200) peak is 2.375 .

Nanocrystals with an icosahedral shape will tend to preferentially lie on a flat substrate on their (111) faces. This increases the diffraction intensity from these planes. However, when compared to gold and palladium nanocrystals with similar icosahedral shapes, the intensity ratios are quite similar (2.69 (ref. 35) and 2.72 (ref. 36), respectively). This indicates that the diffraction from the (111) planes, which is the magnetic easy axis for an fcc Ni crystal, was enhanced for the icosahedra owing to texturing effects.

The formation of the nickel nanocrystals most likely begins with a reduction process. Ethylene glycol has been reported to act as a reducing agent as well as a solvent in polyol reactions, first reported by Fievet and co-workers. ${ }^{37}$ However, as sodium
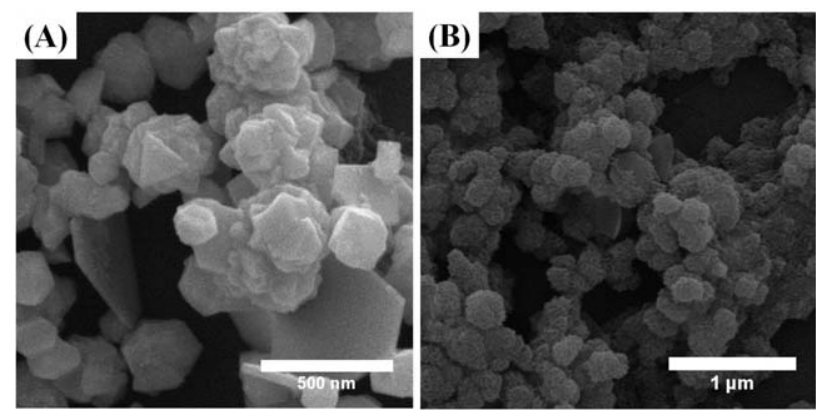

Fig. 5 SEM images of nickel particles synthesised using nickel acetylacetonate as the metal precursor, with a hold time of 10 minutes; using (a) $3 \times 10^{-4} \mathrm{~mol}$ and (b) $9 \times 10^{-4} \mathrm{~mol}$ of sodium formate.

formate is employed as a reducing agent in this reaction, it is unlikely that the nickel nanocrystals will form via this process. Nickel nuclei will be formed by the reduction of the metal precursor by the sodium formate. As is observed in Fig. 5(a), when the amount of sodium formate in the reaction solution was increased from $1.5 \times 10^{-4}$ to $3 \times 10^{-4} \mathrm{~mol}$, the uniformity in the shape of the nanocrystals was greatly diminished. At this concentration of sodium formate, facetted nickel nanoparticles were observed, but the crystalline nature of the particles was reduced when compared to samples synthesised using a lower formate concentration. Upon increasing the amount of sodium formate to $9 \times 10^{-4} \mathrm{~mol}$, no single crystals were formed, as can be seen in Fig. 5(b). Thus, the ratio of ethylene glycol to sodium formate is extremely important in the synthesis of metal nanocrystals with similar shapes and sizes.

The interaction of surfactants or capping ligands with metal nuclei in a solution-phase synthesis will often alter the order of free energies on the metal surface. The formation of a particular morphology of metal nanocrystal will thus be dependent on the relative growth rates of different facets. ${ }^{16}$ Trioctylphosphine oxide (TOPO) was used as a capping agent in the synthesis of the nickel nanocrystals. Puntes et al. have shown that TOPO acts as a selective absorber which alters the relative growth rates of different faces of crystals, synthesising nanostructures with various morphologies. ${ }^{38} \mathrm{TOPO}$ is easily attached to the surface of the nickel nuclei via its $\mathrm{P}=\mathrm{O}$ group. This attachment controls the rate of growth of the nickel nanocrystals. As different facets of nanocrystals have different adsorption and desorption properties, it is expected that facets with fewer attached molecules will grow faster than those with more. As defined in Langmuir's adsorption theorem, a molecule cannot adsorb on a surface where the adsorption sites are already occupied by other molecules. ${ }^{39}$ Thus, the growth rate of the metal nanocrystals can be controlled by the sticking probability of the capping ligands on a given facet of the crystal. Theoretical measurements by Cleveland and Landman indicate that the multiple twinned icosahedron is the most thermodynamically stable seed, as it is bounded almost entirely by $\{111\}$ facets. ${ }^{40}$ The $\{111\}$ face of an fcc metal structure has the lowest surface energy compared to the $\{110\}$ and $\{100\}$ faces $(\{110\}>\{100\}>\{111\})$. This implies that an fcc metal is more likely to nucleate and grow into nanocrystals with their surfaces consisting of the $\{111\}$ facets than the other facets. Thus, in the early stages of the reaction, it is likely that the nuclei formed are kinetic structures with low-energy $\{111\}$ faces. 
Thus, by careful control of the growth rate of the metal nuclei, using sodium formate and ethylene glycol, and controlling the growth of crystal faces using TOPO, multiple twinned nanocrystals are formed rather than single crystal seeds.

\section{Magnetic characterisation}

The magnetic characterisation of two of the nickel nanocrystal samples was investigated by measuring both the hysteresis loops (magnetisation $(M)$ plotted against field strength $(H)$ ) and temperature dependent magnetisation ( $M$ plotted against temperature $(T)$ ) curves. These samples were nickel nanocrystals synthesised with a hold time of 5 and 10 minutes. For each sample, a hysteresis loop was measured at various temperatures from 300 to $5 \mathrm{~K}$, at applied field strengths up to $30 \mathrm{kOe}$. The particle size, coercivity and saturation magnetization values for these samples at 300 and $5 \mathrm{~K}$ are shown in Table 1 . Room temperature hysteresis loops $(300 \mathrm{~K})$ for the samples at applied field strengths between +5000 Oe and -5000 Oe are shown in Fig. 6. Both samples displayed typical ferromagnetic behaviour at room temperature.

Fig. 7 shows the saturation magnetisation values for the two samples at temperatures from 300 to $5 \mathrm{~K}$. Both samples exhibited ferromagnetic behaviour, displaying hysteresis and remanence across the entire temperature range. The saturation magnetisation of these samples was also only weakly dependent on temperature, which is typical of pure nickel samples at temperatures well below their Curie temperature $(631 \mathrm{~K})$. As shown in Fig. 7, the saturation magnetisation $\left(M_{\mathrm{s}}\right)$ of both samples increases with decreasing temperature, as the thermal agitation of the atoms is reduced; a similar variation is seen for bulk nickel. ${ }^{41}$ In comparing the magnitude of the room temperature $M_{\mathrm{s}}$ values of the samples with that of bulk nickel (54.39 $\mathrm{emu} \mathrm{g}^{-1}$ at room temperature ${ }^{42}$ ), the nickel nanocrystals were observed to have room temperature $M_{\mathrm{s}}$ values of 79 and $85 \%$ that of bulk nickel for the 5 and 10 minute hold times respectively. Possible reasons for the smaller than expected $M_{\mathrm{s}}$ values have been previously reported in the literature. ${ }^{43}$ Reductions in $M_{\mathrm{s}}$ can be explained by amorphous non-magnetic structures or the presence of non-magnetic interfaces. Further reasons include electron exchange between ligand and surface atoms which may quench the moment or small amounts of surfactant molecules adsorbed onto the nickel nanoparticle surface.

The variation in the coercivity with temperature for the nickel samples is shown in Fig. 8. Coercivity is an important material property used in the characterisation of magnetic nanoparticles as it will exhibit a very strong and well-established size effect. ${ }^{44} \mathrm{~A}$ series of field-cooled (FC) and zero-field-cooled (ZFC) measurements were carried out on the nickel nanocrystals. In order to obtain the ZFC measurements, the sample was

Table 1 Coercivity $\left(H_{\mathrm{c}}\right)$ and saturation magnetisation $\left(M_{\mathrm{s}}\right)$ values at different temperatures for the samples synthesised

\begin{tabular}{lllll}
\hline Sample & $\begin{array}{l}H_{\mathrm{c}}, 300 \mathrm{~K} / \\
\mathrm{Oe}\end{array}$ & $\begin{array}{l}H_{\mathrm{c}}, 5 \mathrm{~K} / \\
\mathrm{Oe}\end{array}$ & $\begin{array}{l}M_{\mathrm{s}}, 300 \mathrm{~K} / \mathrm{emu} \\
\mathrm{g}^{-1}\end{array}$ & $\begin{array}{l}M_{\mathrm{s}}, 5 \mathrm{~K} / \mathrm{emu} \\
\mathrm{g}^{-1}\end{array}$ \\
\hline $\mathrm{Ni}(\mathrm{acac})_{2}, 5 \min 164$ & 342 & 43.1 & 48.9 \\
$\mathrm{Ni}(\mathrm{acac})_{2}, 10 \quad 147$ & 292 & 46.4 & 51.2 \\
$\quad$ min & & & \\
\hline
\end{tabular}
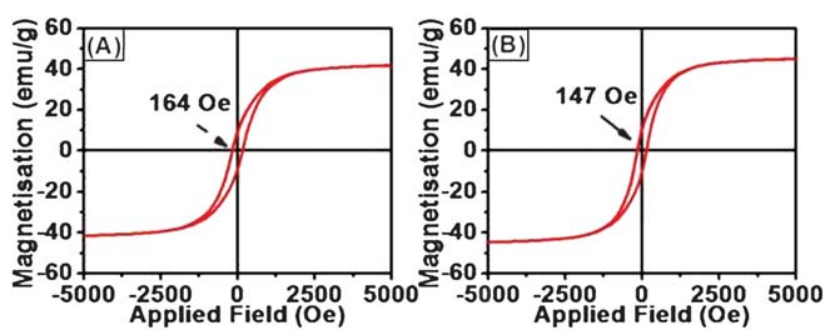

Fig. 6 Room temperature (300 K) hysteresis loops for samples prepared with the nickel acetylacetonate precursor with a microwave hold time of (a) $5 \mathrm{~min}$ and (b) $10 \mathrm{~min}$. Coercivity values for the samples are shown in the graphs.

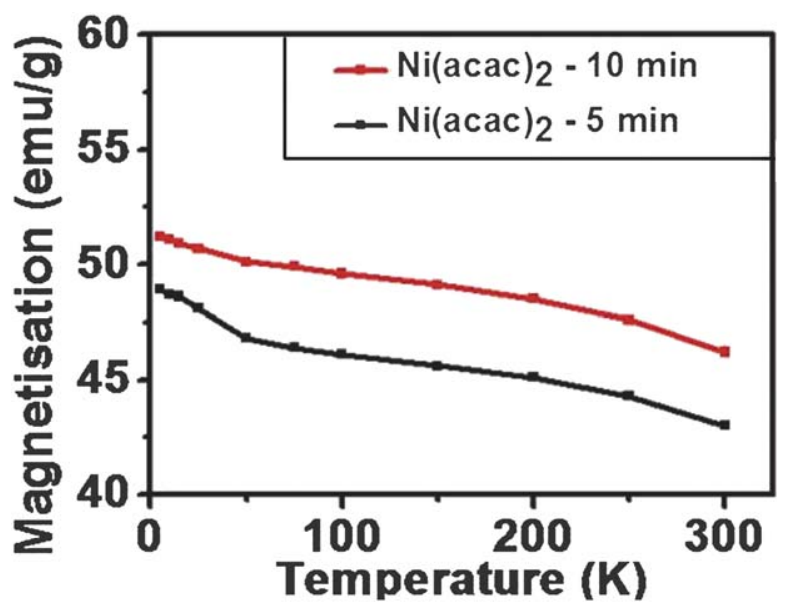

Fig. 7 Magnetisation versus temperature for nanocrystal samples prepared using nickel acetylacetonate with varying hold times.

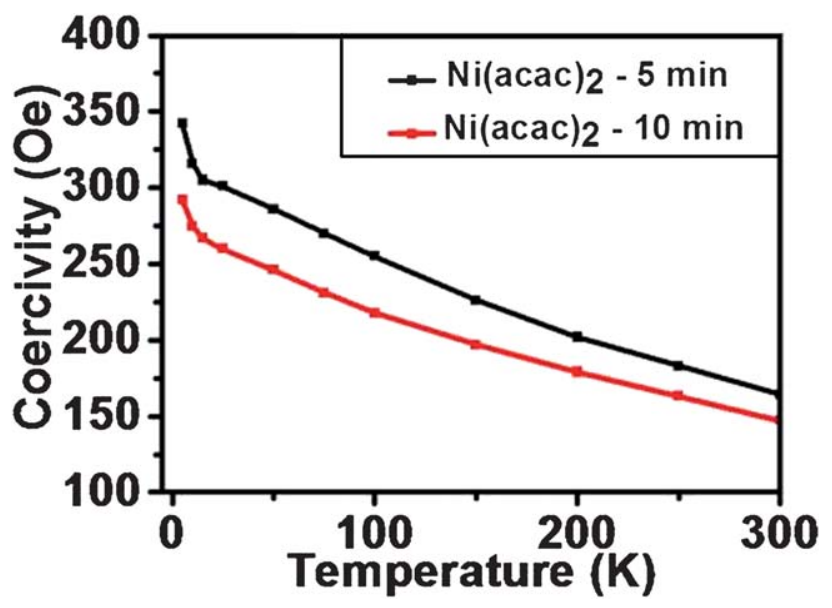

Fig. 8 Measurement of sample coercivity versus temperature for nanocrystal samples prepared using nickel acetylacetonate with varying holdtimes.

completely demagnetised before being cooled to $5 \mathrm{~K}$ in the absence of an external field. An external magnetic field was then applied to the sample and the sample moment was measured as the temperature was continually increased. The FC measurement was taken immediately after the ZFC measurement. In the FC measurement, the moment of the sample was measured as the 


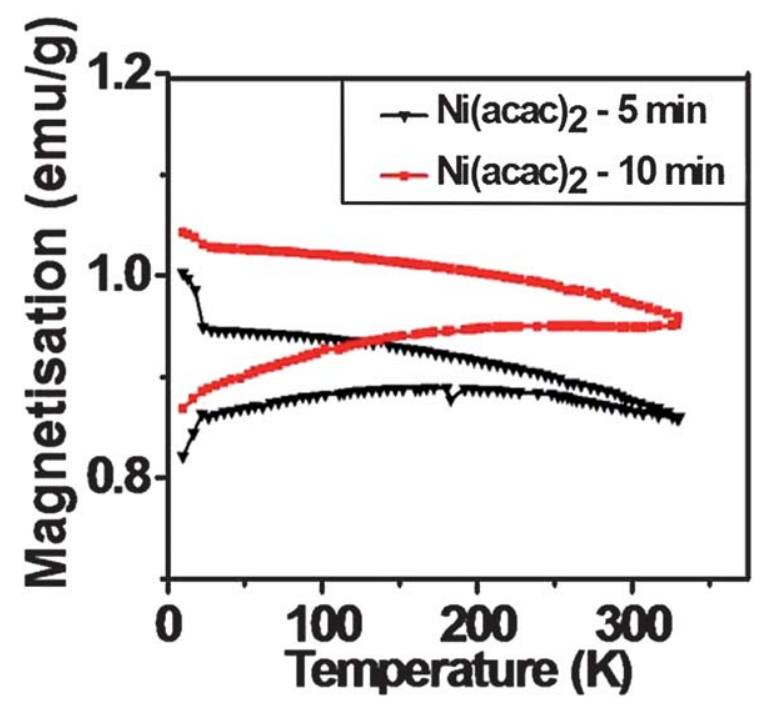

Fig. 9 Field-Cooled (FC) and Zero-Field-Cooled (ZFC) measurements taken with $10 \mathrm{Oe}$ of magnetic field strength applied. The lower portion of each of the curves represents the ZFC measurement and the upper portion represents the $\mathrm{FC}$ measurement.

sample was continuously cooled from the maximum temperature reached in the zero-field-cooled measurement to $5 \mathrm{~K}$ in the presence of the same magnetic field.

Fig. 9 displays the results of these measurements for the two samples. For both samples, the curves are seen to be divergent at the maximum temperature of $330 \mathrm{~K}$ reached in the measurement. This divergence at maximum measurement temperature suggests the presence of large particles, which have been confirmed by SEM analysis. Small single domain particles would display a blocking temperature (the temperature below which the particles would be stable) below room temperature. The relatively flat nature of the FC curves also suggests a degree of dipolar inter-particle interactions which seems likely from the particle's proximity to one another as evident from the TEM and SEM images. ${ }^{45}$

While varying single domain sizes of $\mathrm{Ni}$ (below which the particle becomes a single, uniformly magnetised magnetic domain, as the energy to create and sustain domain walls is no longer favourable) have been reported to be $55 \mathrm{~nm}^{9}$ and $43 \mathrm{~nm},{ }^{46}$ these sizes cannot be taken as absolute, as variations can occur owing to such factors as particle anisotropy. While a critical radius below which a particle is single domain has been reported, provided $K_{\mathrm{u}}$ is large, as:

$$
r_{\mathrm{c}} \approx 9 \frac{\left(A K_{\mathrm{u}}\right)^{0.5}}{\mu_{\mathrm{o}} M_{\mathrm{s}}^{2}}
$$

where $A$ is the exchange stiffness, $K_{\mathrm{u}}$ is the uniaxial anisotropy constant, $\mu_{\mathrm{o}}$ is the permeability of free space and $M_{\mathrm{s}}$ is the saturation magnetisation. ${ }^{47}$ However, this model assumes that the domain wall of a particle has the same structure as that of bulk. These parameters may vary for magnetic particles on the nanometre scale, for example $M_{\mathrm{s}}$ may vary as has previously been discussed. Anisotropy of nanoparticles has also been known to vary as other kinds of anisotropy, such as surface and shape, become more relevant. ${ }^{48}$ In the present study owing to the large sizes of the nanoparticles synthesised, it is likely that the particles may exist in a multi-domain state. The nanocrystal samples synthesised here demonstrate large coercivities at $300 \mathrm{~K}$ when compared to the predicted coercivities of $\mathrm{Ni}$ nano-grains of similar and approaching single domain sizes. On the other hand, isolated multi-domain spherical nanocrystals are predicted to exhibit lower coercive forces than isolated single domain particles. For the multi-domain particles it is therefore likely that their morphology is a possible cause of the large coercivities measured for the samples. These large coercivity values may in turn possibly be attributed to the modified anisotropy of the assembly of nanoparticles into an icosahedral morphology. It is likely that inter-particle interactions too are important in the measured magnetic properties of the samples, with fanning like mechanism (near single domain state) contributing towards magnetisation reversal.

For larger particles, effects other than thermal will have a greater bearing. Bozorth noted that if the dominant anisotropy in a sample of fine particles is crystal anisotropy then an increase in $H_{\mathrm{c}}$ will be expected with decreasing temperature, while the crystal anisotropy of the material also increases rapidly over the same temperature range. ${ }^{49}$ When anisotropy is caused by shape a less rapid change with temperature is to be expected. Parada and Moran reported that for microwave synthesised Ni nanoparticles, different hold times can produce varying proportions of $\mathrm{Ni}$ to $\mathrm{NiO} .^{50}$ The nickel nanocrystal samples synthesised and investigated in our present study were seen to saturate at room temperature discounting the presence of $\mathrm{NiO}$ particles. Compared to the $H_{\mathrm{c}}$ value for bulk nickel, which can reach up to $\sim 40 \mathrm{Oe}^{49}$ depending on the annealing temperature, and to that of nickel nanoplatelets $\left(120 \mathrm{Oe}^{14}\right)$ at room temperature, the nickel nanocrystals synthesised here exhibit an enhanced coercive force, which may be applicable in the areas of high density recording media.

\section{Conclusions}

We report the synthesis of highly faceted, multiply twinned nickel nanoparticles via a microwave-assisted route. Ethylene glycol was employed as the polar solvent, which was necessary for the complete transfer of microwave irradiation throughout the reaction system. Sodium formate was used as a reducing agent and its presence was shown to have a major effect on the overall morphology and shape of the nanocrystals. TOPO was used as the capping ligand. Nickel nanocrystals of icosahedral morphology were synthesised and a mechanism proposed for their agglomeration through the attachment of $\{111\}$ faces. Hysteresis loops measured across a broad range of temperatures demonstrate the ferromagnetic behaviour of the nickel nanocrystals. The coercivity versus temperature measurements suggested that the enhanced magnetic anisotropy may be attributed to the crystals icosahedral morphologies. FC-ZFC curves also suggested a degree of inter-particle interactions which, given the particles proximity as evidenced from SEM analysis, seemed likely.

Due to their shape and magnetic characteristics, these nickel nanocrystals could find potential applications in the areas of catalysis and high-density data storage, respectively. 


\section{Acknowledgements}

The authors acknowledge financial support from Science Foundation Ireland (Project: 06/IN.1/I98). This research was also enabled by the Higher Education Authority Program for Research in Third Level Institutions (2007-2011) via the INSPIRE programme.

\section{References}

1 S. H. Sun, C. B. Murray, D. Weller, L. Folks and A. Moser, Science, 2000, 287, 1989-1992.

2 L. Babes, B. Denizot, G. Tanguy, J. J. Le Jeune and P. Jallet, J. Colloid Interface Sci., 1999, 212, 474- 482.

3 A. G. Roca, R. Costo, A. F. Rebolledo, S. Veintemillas-Verdaguer, P. Tartaj, T. Gonzalez-Carreno, M. P. Morales and C. J. Serna, J. Phys. D: Appl. Phys., 2009, 42, 224002-224012.

4 P. D. Stevens, J. D. Fan, H. M. R. Gardimalla, M. Yen and Y. Gao, Org. Lett., 2005, 7, 2085-2088.

5 Y. Xia, Y. J. Xiong, B. Lim and S. E. Skrabalak, Angew. Chem., Int. Ed., 2009, 48, 60-103.

6 K. L. Kelly, E. Coronado, L. L. Zhao and G. C. Schatz, J. Phys. Chem. B, 2003, 107, 668-677.

7 W. P. McConnell, J. P. Novak, L. C. Brousseau, R. R. Fuierer, R. C. Tenent and D. L. Feldheim, J. Phys. Chem. B, 2000, 104, $8925-8930$.

8 G. Guisbiers, M. Kazan, O. Van Overschelde, M. Wautelet and S. Pereira, J. Phys. Chem. C, 2008, 112, 4097-4103.

9 A. H. Lu, E. L. Salabas and F. Schuth, Angew. Chem., Int. Ed., 2007, 46, 1222-1244.

10 N. Cordente, M. Respaud, F. Senocq, M. J. Casanove, C. Amiens and B. Chaudret, Nano Lett., 2001, 1, 565-568.

11 C. M. Hangarter, Y. Rheem, B. Yoo, E. H. Yang and N. V. Myung, Nanotechnology, 2007, 18, 7.

12 Y. H. Leng, Y. H. Zhang, T. Liu, M. Suzuki and X. G. Li, Nanotechnology, 2006, 17, 1797-1800.

13 Y. H. Leng, Y. Li, X. G. Li and S. Takahashi, J. Phys. Chem. C, 2007, 111, 6630-6633

14 R. Xu, T. Xie, Y. G. Zhao and Y. D. Li, Cryst. Growth Des., 2007, 7, $1904-1911$.

15 V. L. Nguyen, D. C. Nguyen, T. Hayakawa, H. Hirata, G. Lakshminarayana and M. Nogami, Nanotechnology, 2010, 21 , 035605-035621.

16 Y. C. Yu, Y. X. Zhao, T. Huang and H. F. Liu, Mater. Res. Bull., 2010, 45, 159-164.

17 F. Kim, S. Connor, H. Song, T. Kuykendall and P. D. Yang, Angew. Chem. Int. Ed., 2004, 43, 3673-3677.

18 M. Tsuji, M. Ogino, R. Matsuo, H. Kumagae, S. Hikino, T. Kim and S. H. Yoon, Cryst. Growth Des., 2010, 10, 296-301.

19 L. D. Marks, Rep. Prog. Phys., 1994, 57, 603-649.

20 S. Swaminarayan, R. Najafabadi and D. J. Srolovitz, Surf. Sci., 1994, 306, 367-380.

21 N. M. Rosengaard and H. L. Skriver, Phys. Rev. B: Condens. Matter, 1993, 47, 12865-12873.
22 C. L. Lee, C. C. Wan and Y. Y. Wang, Adv. Funct. Mater., 2001, 11, 344-347.

23 J. Park, E. Kang, S. U. Son, H. M. Park, M. K. Lee, J. Kim, K. W. Kim, H. J. Noh, J. H. Park, C. J. Bae, J. G. Park and T. Hyeon, Adv. Mater., 2005, 17, 429-434.

24 L. D. Pachon, M. B. Thathagar, F. Hartl and G. Rothenberg, Phys. Chem. Chem. Phys., 2006, 8, 151-157.

25 V. Tzitzios, G. Basina, M. Gjoka, V. Alexandrakis, V. Georgakilas, D. Niarchos, N. Boukos and D. Petridis, Nanotechnology, 2006, 17, $3750-3755$

26 C. O. Kappe, Chem. Soc. Rev., 2008, 37, 1127-1139.

27 H. Katsuki and S. Komarneni, J. Am. Ceram. Soc., 2001, 84, 23132317.

28 Y. Zhao, J. J. Zhu, J. M. Hong, N. S. Bian and H. Y. Chen, Eur. J. Inorg. Chem., 2004, 4072-4080.

29 K. Okitsu, K. Sharyo and R. Nishimura, Langmuir, 2009, 25, 77867790.

30 A. Lagashetty, V. Havanoor, S. Basavaraja, S. D. Balaji and A. Venkataraman, Sci. Technol. Adv. Mater., 2007, 8, 484-493.

31 Y. K. Hwang, J. S. Chang, Y. U. Kwon and S. E. Park, Microporous Mesoporous Mater., 2004, 68, 21-27.

32 G. Keglevich, T. Novak, L. Vida and I. Greiner, Green Chem., 2006, 8, 1073-1075.

33 S. Navaladian, B. Viswanathan, T. K. Varadarajan and R. P. Viswanath, Nanotechnology, 2008, 19, 045603-045609.

34 J. Berlan, Radiat. Phys. Chem., 1995, 45, 581-589.

35 J. Xu, S. Y. Li, J. Weng, X. F. Wang, Z. M. Zhou, K. Yang, M. Litt, X. Chen, Q. Cui, M. Y. Cao and Q. Q. Zhang, Adv. Funct. Mater., 2008, 18, 277-284.

36 Y. X. Chen, B. L. He, T. Huang and H. F. Liu, Colloids Surf., A, 2009, 348, 145-150.

37 F. Fievet, J. P. Lagier, B. Blin, B. Beaudoin and M. Figlarz, Solid State Ionics, 1989, 32-33, 198-205.

38 V. F. Puntes, D. Zanchet, C. K. Erdonmez and A. P. Alivisatos, J. Am. Chem. Soc., 2002, 124, 12874-12880.

39 I. Langmuir, J. Am. Chem. Soc., 1916, 38, 2221-2295.

40 C. L. Cleveland and U. Landman, J. Chem. Phys., 1991, 94, 73767396.

41 B. D. Cullity and C. D. Graham, Introduction to Magnetic Materials, A Wiley and Sons, Inc., 2009.

42 H. T. Zhang, G. Wu, X. H. Chen and X. G. Qiu, Mater. Res. Bull., 2006, 41, 495-501.

43 D. H. Chen and C. H. Hsieh, J. Mater. Chem., 2002, 12, 2412-2415.

44 G. Herzer, Handbook of Magnetic Materials, Elsevier Science B.V., 1997.

45 J. F. Godsell, K. P. Donegan, J. M. Tobin, M. P. Copley, F. M. F. Rhen, D. J. Otway, M. A. Morris, T. O'Donnell, J. D. Holmes and S. Roy, J. Magn. Magn. Mater., 2010, 322, 1269-1274.

46 W. Gong, H. Li, Z. G. Zhao and J. C. Chen, J. Appl. Phys., 1991, 69, $5119-5121$

47 R. C. O'Handley, Modern Magnetic Materials: Principles and Applications, A Wiley-Interscience Publication, 2000.

48 J. K. Vassiliou, V. Mehrotra, M. W. Russell, E. P. Giannelis, R. D. McMichael, R. D. Shull and R. F. Ziolo, J. Appl. Phys., 1993, 73, 5109-5116.

49 R. M. Bozorth, Ferromagnetism, IEEE Press, 1951.

50 C. Parada and E. Moran, Chem. Mater., 2006, 18, 2719-2725. 